\title{
Erratum: Dispersive optical detection of magnetic Feshbach resonances in ultracold gases [Phys. Rev. A 96, 022705 (2017)]
}

\author{
Bianca J. Sawyer, Milena S. J. Horvath, Eite Tiesinga, Amita B. Deb, and Niels Kjærgaard $\odot$
}

(Received 13 February 2022; published 25 February 2022)

DOI: 10.1103/PhysRevA.105.029901

There is an error in the experimentally measured two-body loss rate coefficients $K_{21}$ throughout the paper which are too small by an exact factor of 2 . This resulted from the measurements being conducted with the atoms distributed over two traps whereas the number of atoms $N \simeq 2.3 \times 10^{6}$ stated is the total number in both traps. The error has been corrected in Ref. [1]. In particular, we note that the correction modifies panel (c) of Fig. 4 of our original paper to appear as shown in Fig. 1 below. In addition, Eq. (B3) of the original paper should read

$$
n_{0}=\frac{N_{2}}{\pi^{3 / 2} \sigma_{x} \sigma_{y} \sigma_{z}},
$$

and the peak density stated in Sec. II C should be corrected to $n_{0} \simeq 2.8 \times 10^{19} \mathrm{~m}^{-3}$.
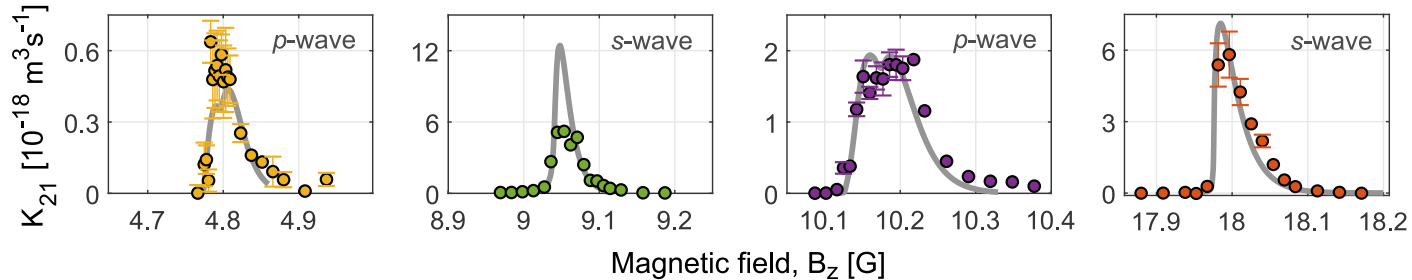

Magnetic field, $B_{z}[G]$

FIG. 1. Theoretically predicted $K_{21}$ coefficients (gray lines), alongside the corresponding measured $K_{21}$ coefficients corrected for the factor of 2 error occurring in the original paper.

[1] B. J. Sawyer, Dispersive probing of dynamical processes and Feshbach resonances in ultracold gases, Ph.D. thesis, University of Otago, 2021. 